\title{
QUADERNI Quaderni
}

Communication, technologies, pouvoir

82 | Automne 2013

L'hôpital à l'épreuve de la performance économique

\section{Gabrielle Hecht, Being Nuclear: Africans and the Global Uranium Trade}

\section{Richard Marion}

\section{(2) OpenEdition}

Journals

Édition électronique

URL : https://journals.openedition.org/quaderni/751

DOI : $10.4000 /$ quaderni.751

ISSN : 2105-2956

Éditeur

Les éditions de la Maison des sciences de l'Homme

\section{Édition imprimée}

Date de publication : 5 octobre 2013

Pagination : 105-108

\section{Référence électronique}

Richard Marion, « Gabrielle Hecht,Being Nuclear: Africans and the Global Uranium Trade », Quaderni [En ligne], 82 | Automne 2013, mis en ligne le 05 octobre 2015, consulté le 08 mars 2022. URL : http:// journals.openedition.org/quaderni/751 ; DOI : https://doi.org/10.4000/quaderni.751 


\section{Compte rendu}

\section{Being Nuclear: Africans and the Global Uranium Trade}

\section{Gabrielle Hecht}

The MIT Press, Cambridge (Massachusets, USA) \& London, 2012

par Richard Marion

Université de Lausanne, Institut des sciences sociales, Centre franco-chinois de sociologie de recherche sur les organisations Université Sun Yatsen de Canton

En se demandant ce que peut concrètement signifier l'adjectif voire le substantif " nucléaire », Gabrielle Hecht offre avec Being nuclear une contribution importante et documentée aux études sociales des sciences et des techniques. En historienne, l'auteure nous propose d'inscrire la production de la notion de nucléaire dans le temps et d'y rechercher la pertinence d'une lecture continuiste face à un discours commun de rupture radicale (postHiroshima) répandu jusque dans les milieux académiques.

Par un travail de déconstruction-reconstruction de l'objet du « nucléaire », Gabrielle Hecht introduit à la pluralité concrète, avant tout matérielle, des lieux de façonnage et d'interprétation de cette notion. Sa recherche de terrain nous plonge ainsi dans la profondeur des mines d'uranium et au cœur des négociations des agences internationales de régulation de l'énergie nucléaire, en passant par les bureaux des décideurs industriels. Il n'y a donc pas une mais bien des nucléarités, concept proposé par l'auteure pour décrire les conflits de labellisation nucléaire qui se déploient en des lieux, à travers des objets matériels et via des contingences loin de tout déterminisme culturel, politique ou même technique.

Gabrielle Hecht s'attache ainsi dans la première partie de l'ouvrage à montrer comment une conception particulariste du nucléaire par les États constitue un 
enjeu de pouvoir. C'est en effet en fonction des perceptions par les États de leur intérêt souverain que se concrétisent - et s'affrontent - précisément les conceptions de ce qui est nucléaire. À travers la façon dont les mécanismes des marchés (de l'extraction du minerai à la consommation de l'énergie) sont mobilisés et (re)configurés, l'auteur montre que la définition de ce qui est nucléaire et la plus ou moins grande spécificité accordée à cette nucléarité fluctuent. C'est donc une question processuelle et située bien davantage qu'une nature déterminée et substantielle. D'une certaine manière, Gabrielle Hecht nous invite ici à une sociologie très politique de l'usage de l'outil et de la dénomination nucléaire.

Poursuivant ce mouvement, la chercheuse ouvre la boîte noire du travail de production de cette qualité de nucléaire. Que la dimension politique et spécifique du nucléaire ait été impulsée par des acteurs étatiques n'empêche en effet pas que cela se concrétise, se traduise et se transforme dans les laboratoires, les bureaux techniques et les mines. Ce que l'auteure appelle le « travail nucléaire » regroupe ainsi toutes les activités contribuant au processus de production de la nucléarité. N'abandonnant pas les dynamiques relevées au cours de la première partie de l'ouvrage, Gabrielle Hecht traduit in concreto les enjeux de labellisation par un processus de mise en visibilité - invisibilité d'une nucléarité instable, socialement voire historiquement réversible. Il s'agit ici de pister les stratégies de gestion de la mise à l'agenda des acteurs intéressés. Ne parvenant pas à banaliser la notion de nucléarité, les industriels de la filière nucléaire déplacent la problématique et travaillent à dénucléariser - donc à banaliser - leur objet ou leur activité. La souplesse de cette stratégie permet ainsi de techniciser les termes du débat éthique et politique tout en demeurant compatible avec un discours rassurant et s'employant à réaffirmer le sérieux avec lequel il conviendrait de traiter tout ce qui relèverait vraiment du nucléaire.

Pluriel et mouvant au gré de conflits symboliques, le nucléaire - civil comme militaire - n'en constitue pas moins un tout, un réseau mondial unifié dont chaque entité participe, joue et dépend in fine. Gabrielle Hecht montre comment industriels, gouvernements, travailleurs et ONG se trouvent tous enrôlés dans une «technopolitique » mondiale traversant les catégories d'analyses comme les espaces ou encore les pratiques, et redéfinissant les intérêts. La puissance par et sur le nucléaire, réside ainsi dans la capacité à stabiliser à son avantage un réseau sociotechnique. Cela prend des 
formes verticales - arrangements (post)coloniaux des anciennes métropoles européennes avec les nouveaux États africains - et plus horizontales - cartel des acheteurs de minerais d'uranium, stabilisant une forme d'interdépendance par des échanges d'informations permettant une observation réciproque.

Suivant notamment la circulation de l'uranium de par le monde et les différents programmes de recherche, Gabrielle Hecht jette un regard nouveau sur ce qui est souvent considéré comme une affaire de technique, dont la puissance exceptionnelle justifierait, philosophiquement et politiquement, une rupture avec les normes et institutions du monde précédant l'explosion de la première bombe atomique en 1945. Une partie de la science politique et l'étude des relations internationales de la Guerre froide présentent ainsi un exceptionnalisme nucléaire s'imposant en bloc. Sans rejeter cette portée et cette traduction possibles des utilisations militaires de l'énergie nucléaire, l'auteure préfère les recontextualiser dans une dynamique sociale co-constructrice des technologies nucléaires, les dimensions matérielles constituant le point d'appui initial de son enquête, la pointe émergée de l'iceberg qu'elle cherche à montrer. Donnant la priorité à la matérialité du terrain investigué, l'analyse monte ensuite en abstraction pour aborder la dynamique symbolique et politique en négociation à tous les niveaux. C'est en fin de compte non seulement l'usage de l'énergie nucléaire - civile, militaire ou duale - mais aussi le travail de production technique du nucléaire qui se trouve indissociable de ses enjeux sociaux et politiques. Gabrielle Hecht parle ainsi de «nucléarité » non comme propriété essentielle mais bien comme construction sociotechnique. C'est dans cet esprit que l'auteure part dans la seconde partie de son ouvrage sur les traces de la production de l'énergie nucléaire en remontant aussi loin qu'il lui est matériellement possible : au niveau du travail d'extraction du minerai.

Construisant un second récit de la filière nucléaire - qui ne tardera pas à rejoindre le premier - l'auteure vient donc à la rencontre des hommes et des femmes qui par leur travail donnent une traduction matérielle à l'existence et à la nucléarité de l'uranium. C'est peut-être ici que l'ouvrage se montre le plus convaincant et le plus novateur pour la littérature académique ; l'analyse la plus matériellement ancrée « reconstruit » en effet les niveaux les plus macro de la politique internationale à partir du jeu des acteurs de terrain. $\mathrm{La}$ plongée au cœur des mines et usines du nucléaire rejoint et reconsidère ainsi les réseaux internationaux décrits dans la première partie. 
Ayant déconstruit l'évidence de la stabilité et la neutralité politique de l'exceptionnalisme nucléaire à partir du cœur même de sa formation statocentrée, Gabrielle Hecht n'en reste pas là car, à l'écoute de ces récits, elle comprend bien que se pratique un jeu de la réversibilité et de l'intermittence des qualificatifs utilisés, instrumentés. Plus précisément, c'est-à-dire plus matériellement, il s'agit de la production stratégique par les acteurs de la filière nucléaire de régimes de visibilité et d'invisibilité.

La chercheuse n'en reste pas là dans les nouvelles perspectives qu'elle nous propose sur les objets du nucléaire : Gabrielle Hecht démontre aussi que même une grille de lecture en termes de dominants et de dominés n'amène pas nécessairement à catégoriser ces acteurs a priori car ce qui est dominé ici ne l'est peut-être pas là-bas et ne le sera pas forcément demain. L'historienne démêle, déroule et nous présente ainsi sous plusieurs angles les multiples fils d'une pelote que nul n'a pu complètement sectionner.

Derrière la modestie de ses conclusions, c'est donc bien d'un unique réseau mondial de l'uranium dont nous parle ici Gabrielle Hecht. Dans quelle mesure cet ensemble d'acteurs du nucléaire constitue-t-il un acteur-réseau au sens de Michel Callon ? Comment les nouvelles remises en cause de la légitimité politique de l'énergie nucléaire affectent-elles ou précipitent-elles une telle constitution en acteur réseau?

La lecture d'une étude empirique aussi serrée et concluante invite plus généralement à se poser la question de débouchés théoriques pour les études sociales des sciences et des techniques, voire à se demander pourquoi ne pas construire une théorie à partir de cet ancrage de terrain. Reposant sur une analyse aussi précise que concrète, le travail de l'auteure remonte et reconstruit en effet opportunément les acteurs macrosociologiques en les ancrant dans une dynamique et une articulation microsociologiques toujours finement situées. Ainsi semblent réunies les conditions d'une montée en généralité sans encombrants présupposés théoriques et sans lâcher les acteurs, traducteurs à tous les niveaux de ces dynamiques mondiales. 